\title{
An atypical cause of atypical chest pain
}

\author{
Ahmad Zaheen MD, Reed A Siemieniuk MD, Patrick Gudgeon MD
}

A Zaheen, RA Siemieniuk, P Gudgeon. An atypical cause of atypical chest pain. Can J Infect Dis Med Microbiol 2014;25(5):253-254.

The present report describes a case involving a 57-year-old HIVpositive man who presented with acute retrosternal chest pain accompanied by $24 \mathrm{~h}$ of fever. Septic arthritis of the manubriosternal joint was diagnosed based on magnetic resonance imaging findings in addition to Staphylococcus aureus bacteremia. To the authors' knowledge, the present case is only the 12th reported case of manubriosternal septic arthritis, and the first in an HIV-positive patient. Early diagnosis and treatment can circumvent the need for surgical intervention. Based on the present case report and review of the literature, the authors summarize the epidemiology, appropriate imaging and suggestions for antibiotic therapy for this rare presentation.

Key Words: Chest pain; HIV; Manubriosternal septic arthritis

\section{CASE PRESENTATION}

A 57-year-old HIV-positive man was admitted to general internal medicine after presenting with acute-onset retrosternal chest pain accompanied by a $24 \mathrm{~h}$ history of fever. The patient also reported a resolving headache with intermittent nausea. Apart from longstanding HIV infection, his medical history was significant for Charcot-MarieTooth disease with a chronic ulcer on his right first toe, osteoporosis, kyphoscoliosis and depression. Two weeks before presentation, his CD4 count was 233 cells $/ \mathrm{mm}^{3}$ with a viral load of $<40$ copies $/ \mathrm{mL}$. The patient did not use intravenous (IV) drugs and was compliant with all of his medications including antiretroviral therapy (darunavir, ritonavir, maraviroc and raltegravir), alendronate and buproprion.

On examination, the patient was alert, diaphoretic and visibly uncomfortable. He was febrile $\left(39.4^{\circ} \mathrm{C}\right)$, with a blood pressure of $122 / 72 \mathrm{mmHg}$, heart rate of 100 beats $/ \mathrm{min}$, respiratory rate of 24 breaths/min and oxygen saturation of $100 \%$ on room air. His neck was supple; cardiovascular, respiratory and abdominal examinations were unremarkable. His neurological examination was normal and there were no rashes, active joints or stigmata of endocarditis. The patient's chest pain was retrosternal, pleuritic, localized to the manubriosternal joint (MSJ) with some radiation to the scapula and was reproducible by palpation. His foot ulcer showed no obvious signs of infection and there was no history of recent cellulitis. Complete blood count, electrolyte, creatinine and cardiac enzyme levels and a urinalysis were all normal. An electrocardiogram showed sinus tachycardia. His chest $\mathrm{x}$-ray was normal and computed tomography (CT) ruled out pneumonia, fractures, pulmonary embolism and aortic dissection. Twelve hours after admission, two of two peripheral blood cultures were positive for Gram-positive cocci in clusters. Thirty-six hours after admission, the organism was speciated as Staphyloccocus aureus. Transthoracic and transesophageal echocardiography did not detect vegetations suggestive of endocarditis. The patient was started empirically on IV vancomycin, which was changed to cefazolin when

\section{Une cause inhabituelle de douleur thoracique}

Le présent rapport décrit le cas d'un homme de 57 ans atteint du VIH qui a consulté en raison d'une douleur thoracique rétrosternale aiguë accompagnée de fièvre depuis 24 heures. Les résultats de l'imagerie par résonance magnétique ont permis de diagnostiquer une arthrite septique de l'articulation manubrio-sternale, ainsi qu'une bactériémie à Staphylococcus aureus. En autant que le sache les auteurs, il s'agit du $12^{\mathrm{e}}$ cas déclaré d'arthrite septique manubrio-sternale seulement, et le premier auprès d'un patient positif au VIH. Grâce à un diagnostic et un traitement rapides, on peut éviter l'intervention chirurgicale. Compte tenu du présent rapport de cas et de l'analyse bibliographique, les auteurs résument l'épidémiologie, l'imagerie pertinente et les suggestions d'antibiothérapie de cette présentation rare. the antibiotic sensitivities of the $S$ aureus isolate became available $60 \mathrm{~h}$ after admission.

Despite resolution of the patient's fever within $24 \mathrm{~h}$ of the initiation of antibiotics, he continued to experience severe central chest pain. By $48 \mathrm{~h}$ after admission, a new, tender mass was noted to have formed over the patient's MSJ. Inflammatory markers analyzed on the third day of admission were significantly elevated (C-reactive protein $48 \mathrm{mg} / \mathrm{L}$ and erythrocyte sedimentation rate $120 \mathrm{~mm} / \mathrm{h}$ ). Although no obvious pathology of the MSJ was noted on the CT scan of the thorax, further imaging of this area was prompted by the patient's unresolving symptoms. Thoracic magnetic resonance imaging (MRI) six days after admission revealed phlegmonous regions anterior and posterior to the MSJ, with lobulated enhancements that were not initially appreciated on CT (Figure 1). A clearly drainable collection was not identified. These findings were believed to be most consistent with septic arthritis of the MSJ. As a result, the course of IV cefazolin was extended from two to six weeks. A surgical opinion was not sought because the

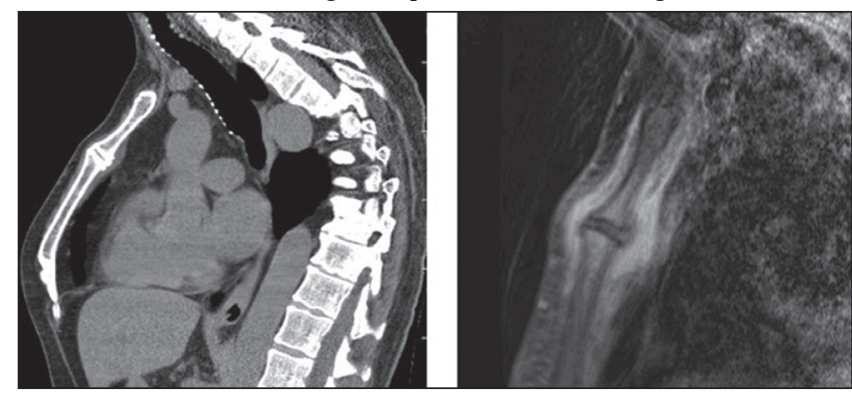

Figure 1) Sagittal views of the thorax in the patient via computed tomography (left) and magnetic resonance imaging (T1 post contrast). No medical abnormalities of the manubriosternal joint were noted on the computed tomography scan, while magnetic resonance imaging shows inflammatory consolidations anterior and posterior to the joint

Department of Medicine, University of Toronto, Toronto, Ontario

Correspondence: Dr Patrick Gudgeon, Trillium Health Centre, 100 Queensway West, Mississauga, Ontario L5B 1B8. Telephone 905-848-7557, e-mail patrick.gudgeon@utoronto.ca 
patient began to improve clinically. He was discharged home after eight days with a peripherally inserted central catheter. On discharge, the patient appeared well, was afebrile and his chest pain had resolved. Three sets of repeat blood cultures were negative. The mass at the MSJ could not be palpated two weeks postdischarge and he remained well four months later.

\section{DISCUSSION}

Acute chest pain is one of the most common reasons for presentation to the emergency department and, in the United States alone, accounts for approximately eight million visits per year (1). Determining the etiology of a patient's chest pain necessitates a broad differential diagnosis because more than one-half of these presentations are due to noncardiac causes (1). To our knowledge, a total of 11 cases of MSJ septic arthritis have been reported previously; the present case is the first to be documented in an HIV-positive patient. The discussion below includes our patient in the summary of cases to date.

The MSJ includes the bony articulation of the manubrium superiorly and the sternum inferiorly. It is a secondary cartilaginous or symphysis joint, which has a lower risk of infection relative to synovial joints (2). Owing to the rarity of this condition, the epidemiology and risk factors for septic arthritis of the MSJ are based on case series, summarized previously in a report by Peng et al (2), with two additional cases (including the present case) having been reported since (3). To date, the majority of patients with MSJ septic arthritis have been male $(75 \%)$, with a mean age of presentation of 49 years (median 46 years; range 20 to 85 years) $(2,3)$. A primary source of bacteremia outside of the MSJ (eg, idiopathic bacteremia or cellulitis) was the most common risk factor and S aureus was isolated in $67 \%$ (eight of 12 ) of cases $(2,3)$. Other reported risk factors include corticosteroid-induced immunosuppression, inflammatory joint disease and IV drug use (2). A more recent report argued that derangements in the bony architecture of the thorax, such as in patients with advanced scoliosis, may transmit to the MSJ and result in joint subluxation and subsequent susceptibility to septic arthritis (3). Interestingly, our patient presented with significant kyphoscoliosis; however, he had no previous history of subluxation or pain at the MSJ and imaging failed to demonstrate signs of anatomical abnormalities at the MSJ. Our patient's MSJ was likely seeded hematogenously secondary to his $S$ aureus bacteremia. It is unclear to what extent the patient's HIV status contributed to his clinical presentation, although HIV infection is a known risk factor for septic arthritis in general (4).

While there are no known pathognomonic findings for MSJ septic arthritis, almost universally, central chest pain is accompanied by a tender and/or palpable mass at the MSJ $(2,3)$. Additionally, fever and leukocystosis are commonly observed, representing a marked inflammatory response. Blood or synovial cultures were positive in $83 \%$ (10 of 12) of reported cases and provided sensitivities to guide antibiotic therapy $(2,3)$. Of those reported, $62 \%$ (five of eight) of blood cultures were positive, compared with $90 \%$ (nine of 10 ) of MSJ cultures. Both blood and MSJ cultures were positive in 67\% (four of six) of cases, when performed $(2,3)$. Imaging aided in the diagnosis of several cases, with MRI suggested as the optimal modality owing to superior visualization of tissue and bone planes at the MSJ (5).

Our patient presented with all of the above signs/symptoms, with the exception of leukocytosis. Diagnostic confirmation was obtained based on positive blood cultures and the MRI findings after a CT scan failed to detect any abnormalities.

Medical therapy with IV antibiotics tailored to culture and sensitivity results (either blood or MSJ aspirate) occurred in almost all cases $(2,3)$. The duration of therapy was typically four to six weeks, consistent with the therapy for other similar forms of septic arthritis $(2,3)$. In all previous cases successfully treated medically (33\%), prompt diagnosis led to early initiation of antibiotics. In the two culture-negative cases, both patients received surgical debridement in combination with broad-spectrum IV antibiotics $(2,5)$. This highlights the importance of blood or synovial cultures in tailoring the antibiotic regimen given the prolonged duration of therapy.

The majority of reported cases $(67 \%)$ required surgical management in addition to antibiotics. Of these cases, three followed a delay in diagnosis while one occurred following failure of antibiotics $(2,3)$. Several surgical cases demonstrated evidence of MSJ destruction, highlighting the urgent need for appropriate treatment to prevent irreparable damage. Similar to the medically treated patients, all surgical cases received a six-week course of IV antibiotics tailored to culture sensitivity results, with good clinical outcomes $(2,3)$. In previous cases of septic arthritis, serial imaging was not reported (2,3). This may only be necessary if there is concern for failure of antibiotic therapy (ongoing fevers, symptoms, worsening joint disruption).

Our patient was managed with six weeks of IV cefazolin based on the sensitivities of the blood culture S aureus isolate. Given his rapid clinical improvement with antibiotics, surgical debridement was not required.

\section{CONCLUSION}

We present a case of MSJ septic arthritis and the first in an HIVpositive patient. Diagnosis was made based on the patient's fever, chest pain, positive blood cultures and MRI findings. The patient was treated with six weeks of IV antibiotics with a good clinical outcome.

DISCLOSURES: The authors have no conflicts of interest to declare.

\section{REFERENCES}

1. Storrow AB, Gibler WB. Chest pain centers: Diagnosis of acute coronary syndromes. Ann Emerg Med 2000;35:449-61.

2. Peng EW, McKillop G, Prasad S, Walker WS. Septic arthritis of the manubriosternal joint. Ann Thorac Surg 2007;83:1190-4.

3. Nwaejike N, Unsworth-White MJ. Manubriosternal subluxation/ dislocation can lead to manubriosternal septic arthritis in patients with kyphoscoliosis. Ann Royal Coll Surg Engl 2010;92:W35-7.

4. Saraux A, Taelman H, Blanche P, et al. HIV infection as a risk factor for septic arthritis. Br J Rheum 1997;36:333-7.

5. Sinha S, Sinha A, Nagarajah K, Oei EL, Critchley P, McNally MA. Chronic sternal osteomyelitis complicating primary manubriosternal septic arthritis. Clin Rheum 2006;25:934-6. 


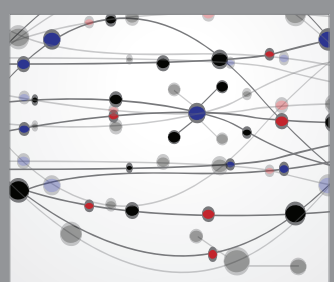

The Scientific World Journal
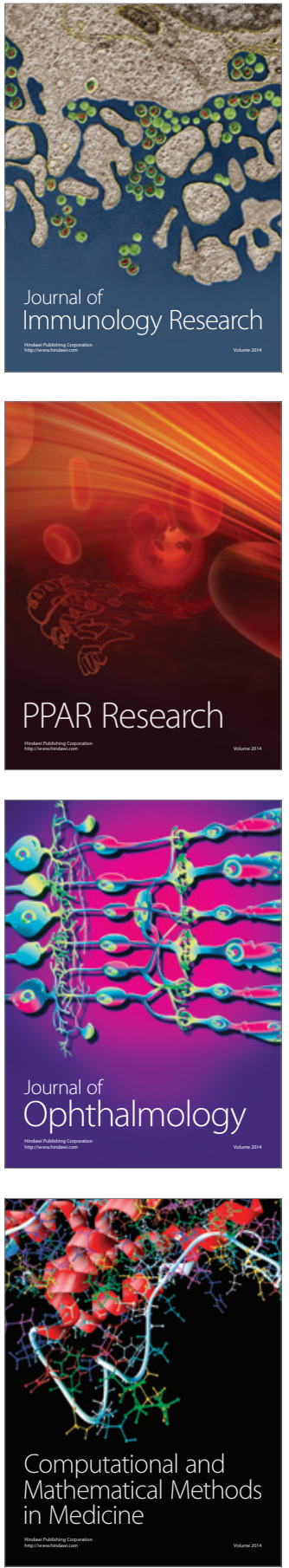

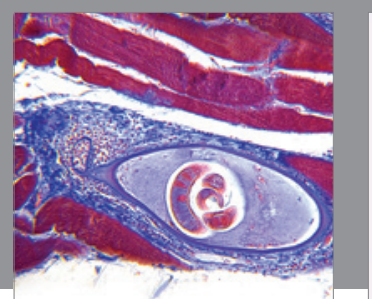

Gastroenterology Research and Practice

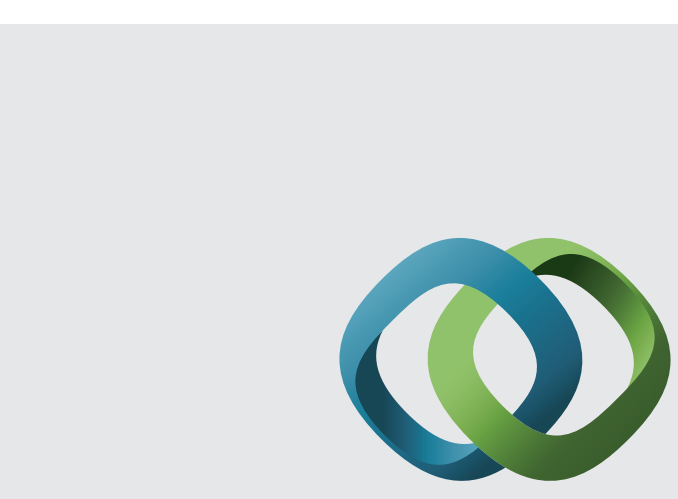

\section{Hindawi}

Submit your manuscripts at

http://www.hindawi.com
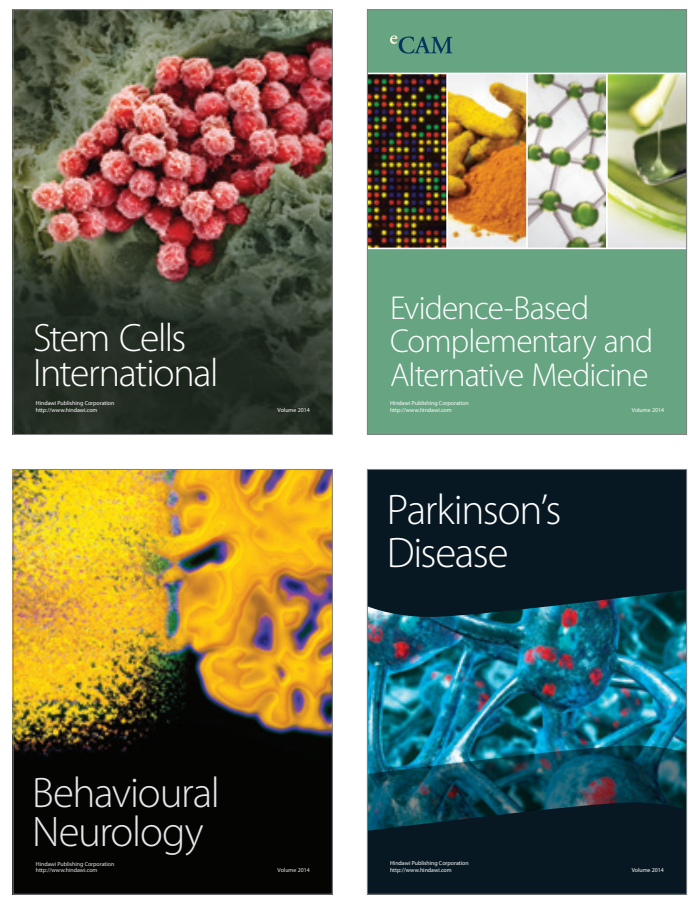
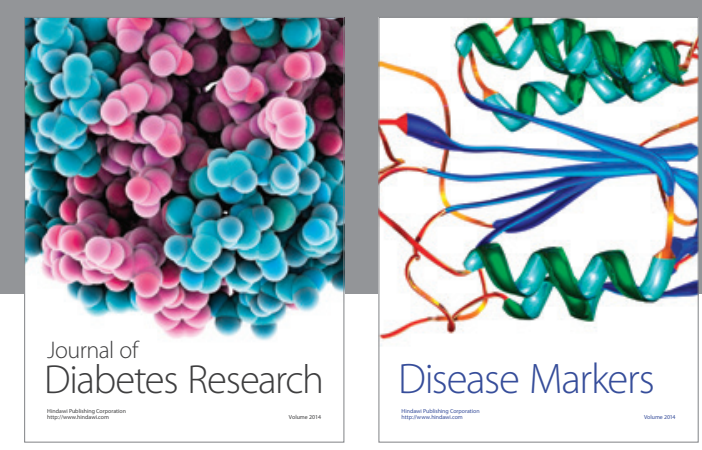

Disease Markers
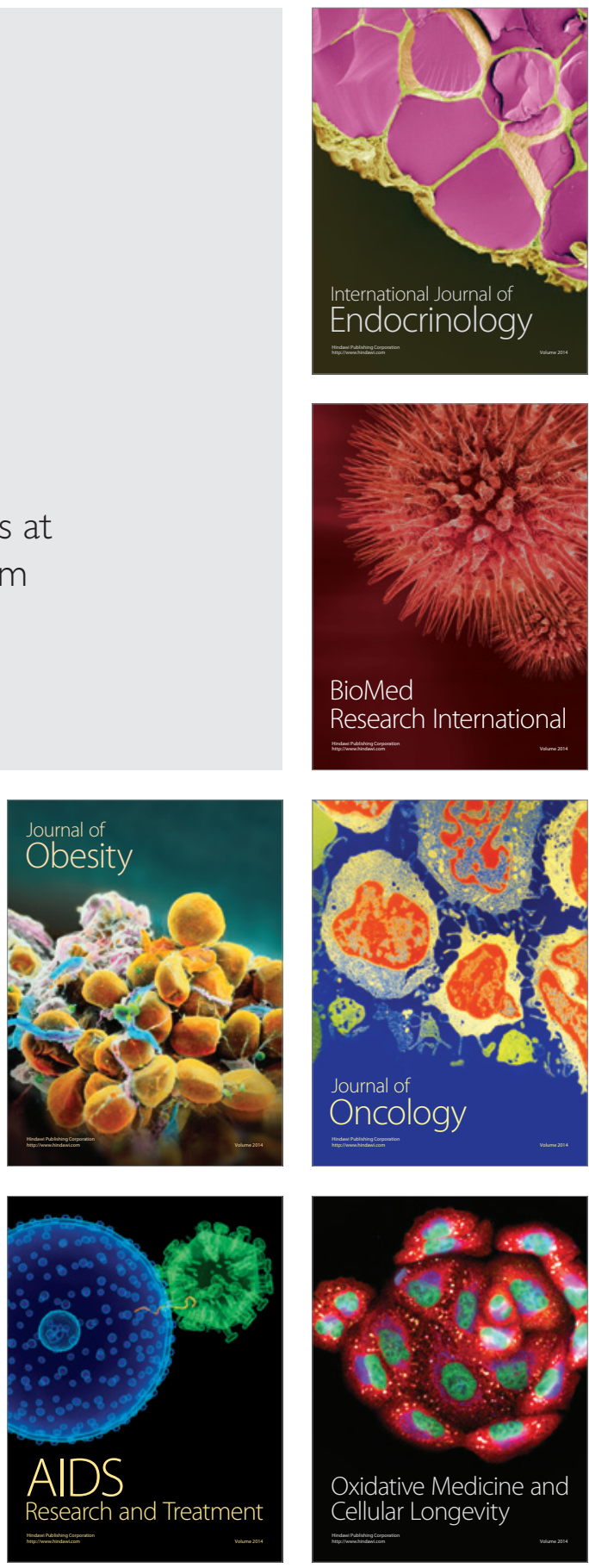